\title{
Measurements of Hadron Production and Under- lying Event Studies at CMS
}

\author{
Danilo Piparo for the CMS Collaboration \\ Karlsruhe Institute of Technology, Kaiserstraße 12, 76131 Karlsruhe, Germany \\ DOI: http://dx.doi.org/10.5689/UA-PROC-2010-09/25
}

\begin{abstract}
Measurements of hadron production, underlying event and forward processes in $p p$ collisions at $\sqrt{s}=0.9,2.36$, and $7 \mathrm{TeV}$, recorded with the CMS detector are presented. Transverse momentum, pseudorapidity and multiplicity distributions of charged hadrons are shown, employing jet triggers to enhance yields at high $p_{T}$. Spectra of identified strange hadrons are characterised. The underlying event is studied measuring the multiplicity and energy density of charged particles in a region perpendicular to the plane of the hard scattering or by exploiting the jet area/median approach. This study allows to discriminate among Monte Carlo models with different multiple parton interactions schemes.
\end{abstract}

\section{The CMS Detector}

The results presented here evaluate the LHC collision data collected with the CMS detector at the centre of mass energies of $\sqrt{s}=0.9,2.36$, and $7 \mathrm{TeV}$. A detailed description of the CMS detector can be found elsewhere [1].

A right-handed coordinate system is used with the origin at the nominal interaction point. The $x$ axis points radially inwards to the center of the LHC ring, the $y$ axis points upwards, and the $z$ axis is parallel to the anti-clockwise beam direction. The azimuthal angle $\phi$ is measured with respect to the $x$ axis in the $x-y$ plane and the polar angle $\theta$ is defined with respect to the $z$ axis.

The CMS pixel and silicon strip tracker plays a crucial role in the results discussed here. The device is immersed in a uniform $3.8 \mathrm{~T}$ magnetic field provided by a $6 \mathrm{~m}$ diameter superconducting solenoid, measures charged particle trajectories in the pseudorapidity range $|\eta|<2.5$, where $\eta=-\ln (\tan (\theta / 2))$. The resolution of the impact parameter is about $100 \mu \mathrm{m}$; the $\mathrm{p}_{T}$ resolution for $1 \mathrm{GeV}$ charged particles is between $0.7 \%$ at $\eta=0$ and $2 \%$ at $|\eta|=2.5[1]$.

\section{Transverse Momentum, Pseudorapidity and Multiplic- ity Distributions of the Charged Hadrons}

An essential first step in exploring a new energy regime of particle collisions [2] is the measurement of particle yields and its kinematic distributions. Such studies contribute to the understanding of the physics of hadron production, including the relative roles of soft and hard scattering contributions. In the complicated environment of LHC $p p$ collisions, the knowledge of the rates and distributions of inclusive particle production is needed to distinguish rare signal 
events from the much larger backgrounds of soft hadronic interactions. The bulk of the particles produced in $p p$ collisions arise from soft interactions, which are described by phenomenological models. Such models must be tuned by the experimental results.

All results presented here, except the ones in section 5 , refer to inelastic non-single-diffractive (NSD) interactions. The count of primary charged hadrons $N_{c h}$ is defined to include decay products of particles with proper lifetime less than $1 \mathrm{~cm}$.

As discussed in [3], CMS provides three methods to measure charged particles, based on counting the following quantities: (i) reconstructed clusters in the barrel part of the pixel detector; (ii) pixel tracklets composed of pairs of clusters in different pixel barrel layers; and (iii) tracks reconstructed in the full tracker volume. The third method also allows for a measurement of the $d N_{c h} / d p_{T}$ distribution.

Figure 1 (left) shows the distributions of $d N_{c h} / d \eta$, averaged over the three measurement methods for three different centre of mass energies at CMS and compared with data from UA5 $(p \bar{p}$, with statistical errors only) and ALICE (with systematic uncertainties). The shaded band shows systematic uncertainties of the CMS data. The CMS and UA5 data are averaged over negative and positive values of $\eta$. Figure 1 (right) shows the average value of $d N_{c h} / d \eta$ in the central $\eta$ region as a function of centre of mass energy. Also shown are NSD and inelastic measurements from the NAL Bubble Chamber $(p \bar{p})$, ISR $(p p)$, UA1 $(p \bar{p})$, UA5 $(p \bar{p})$, CDF $(p \bar{p})$, STAR $(p p)$, PHOBOS $(p p)$ and ALICE $(p p)$. The curves are second-order polynomial fits for the inelastic (solid) and NSD event selections (dashed). The error bars include systematic uncertainties, when available. Data points at 0.9 and $2.36 \mathrm{TeV}$ are slightly displaced horizontally for visibility. A rather steep increase is visible between 0.9 and $7 \mathrm{TeV}$, which is measured to be $[66.1 \pm 1.0($ stat $) \pm 4.2($ syst $)] \%$.
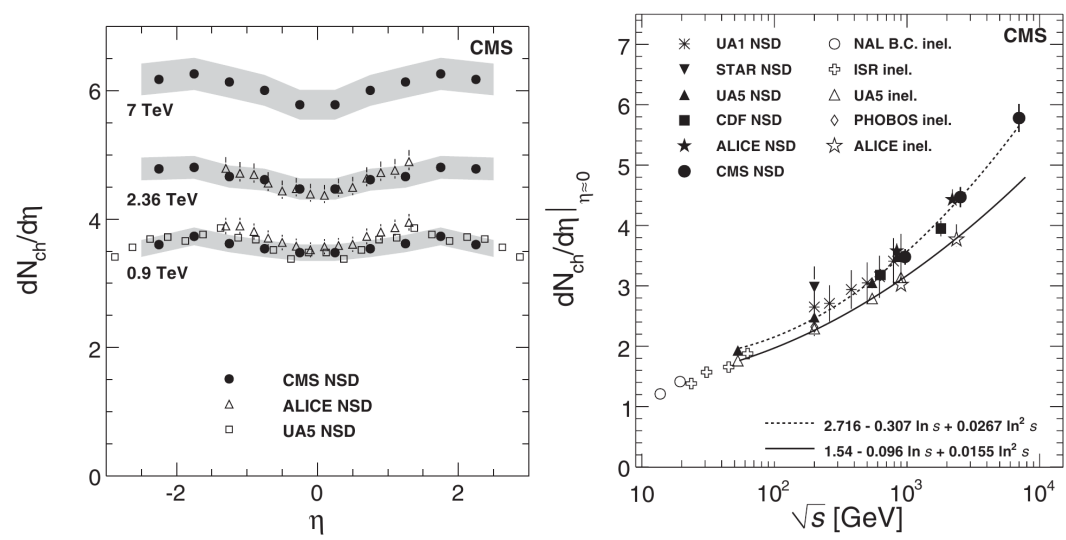

Figure 1: Left: $d N_{c h} / d \eta$ distributions measured at different centre of mass energies. Right: $d N_{c h} / d \eta$ distributions in the central barrel region as a function of the centre of mass energy.

The multiplicity distribution of charged particles has also been measured [4]. Corrections are applied to the data for the trigger and event selection efficiency, for effects of tracking inefficiency, to secondary tracks originating from the decay of long lived particles and to products of interactions with the beam pipe and the detector inactive material. The multiplicity distributions serve as input to improve the Monte Carlo modelling of minimum bias events. Figure 2 (left) shows a comparison of the measurements with three classes of models for charged hadrons 
that have transverse momenta larger than $500 \mathrm{MeV} / \mathrm{c}$. While PYTHIA 8 [5] is the only model that gives a reasonable description of the multiplicity distribution at all energies, it tends to overestimate the transverse momentum spectrum of high multiplicity events. This is further illustrated by the distribution of the average transverse momentum versus $n$ in figure 2 (right).
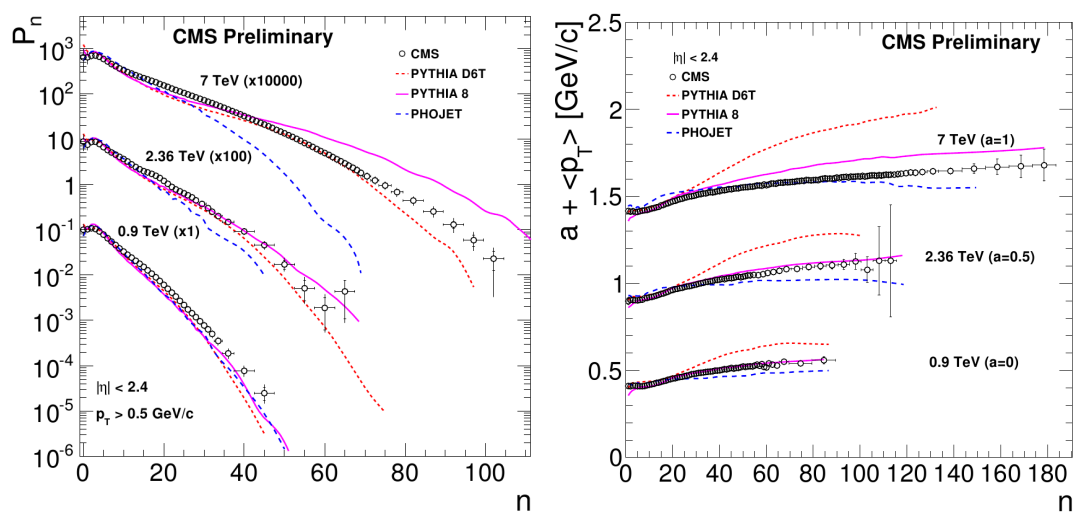

Figure 2: Left: Charged particle multiplicity distributions in $|\eta|<2.4$ and $\mathrm{p}_{T}>500 \mathrm{MeV} / \mathrm{c}$ compared to two different PYTHIA models and the PHOJET [6] model at $\sqrt{s}=0.9,2.36$, and 7.0 TeV. Results for different centre of mass energies are scaled with a multiplicative factor for clarity. Right: Comparison of $\mathrm{p}_{T}$ versus $n$ for $|\eta|<2.4$ with two different PYTHIA models and the PHOJET model at $\sqrt{s}=0.9,2.36$, and $7.0 \mathrm{TeV}$. Results for different energies are shifted for reasons of clarity.

The multiplicity of the charged particles as a function of transverse momentum has also been studied [7]. It is not only the minimum bias events that have been considered here. An enhanced reach of the measured transverse momenta is obtained by exploiting triggers on calorimeter jets (see figure 3). The result obtained with this strategy is found to be compatible with the measurement which considers minimum bias events only in the low transverse momentum region. Except for the excess of tracks at very low transverse momentum compared to common generator tunes, the results are found to be in reasonable agreement with PYTHIA leadingorder pQCD, as well as with the CMS $7 \mathrm{TeV}$ measurement [2]. This result removed a large uncertainty from an important ingredient for $\mathrm{PbPb}$ measurements, namely the $p p$ reference spectrum for the $\mathrm{PbPb}$ collisions at $2.76 \mathrm{TeV}$ per nucleon.

\section{Spectra of Identified Strange Hadrons}

The spectra of strange hadrons have been measured in $p p$ collisions at 0.9 and $7 \mathrm{TeV}$ centre of mass energies [8]. Among the others, the $\Lambda^{0}$ baryons are reconstructed from their decay topologies and the event yield is measured as a function of the rapidity (see figure 4).

The results show an increase in the production rate of strange particles not yet simulated correctly in the current tunes of PYTHIA. The discrepancy gets larger at higher centre of mass energy and for higher mass and strangeness. 

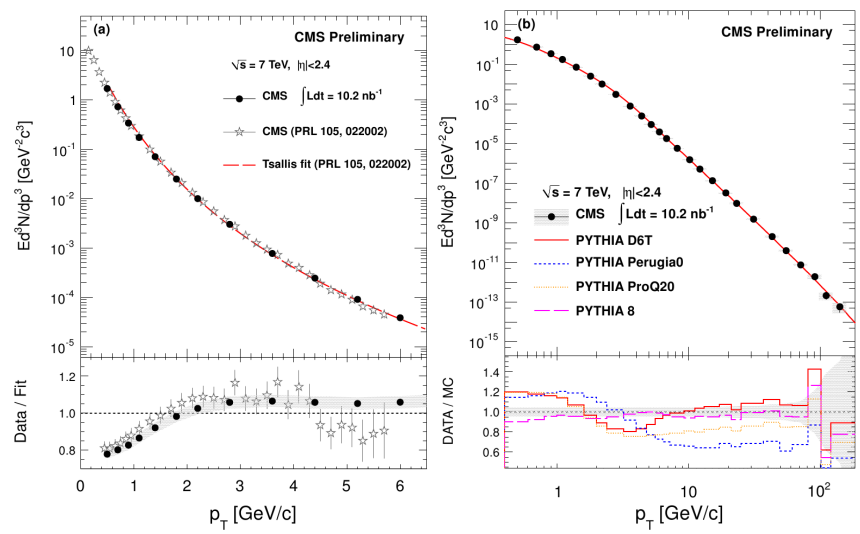

Figure 3: Left, Upper: Invariant differential yield obtained with minimum bias events (stars) and with jet triggers (solid circles) over the limited $\mathrm{p}_{T}$ range accessible with minimum bias events only. Left, Lower: The ratio of the result using jet triggers (solid circles) and minimum bias events (stars) to a fit [2] of the minimum bias only measurement. Right, Upper: Invariant differential yield at $\sqrt{s}=7 \mathrm{TeV}$ compared to PYTHIA D6T (solid red line) over an extended transverse momentum range. Right, Lower: The ratio of the measurement obtained using jet triggers to a number of PYTHIA tunes. The grey band corresponds to statistical plus systematic errors in quadrature.

\section{$4 \quad$ Underlying Event}

In the presence of a hard process, characterised by the presence of particles with large transverse momentum with respect to the beam direction, the final state of proton-(anti)proton interactions can be described as the superposition of several contributions: (i) products of the partonic hard scattering with the highest $\mathrm{p}_{T}$, including initial and final state radiation; (ii) Hadrons produced in additional "multiple parton interactions" (MPI); and (iii) "beam-beam remnants" (BBR) resulting from the hadronisation of the partonic constituents that did not participate in other scatterings. Products of MPI and BBR form the "underlying event" (UE). It should be observed that the UE cannot be uniquely separated from initial and final state radiation.

Two methods for the UE measurements are discussed here. The former, referred as to "traditional" approach, foresees the measurement of multiplicity and energy density in the region transverse to a leading object in the event, such as the leading track or track-jet [9]. The latter, referred as to "jet area/median" approach, does not require a leading object in the event and implies the study of the UE activity in terms of the median $\rho$ (equation 1) of the distribution of the transverse momentum divided by the area $\left(p_{T}^{j e t} / A_{j e t}\right)$ for the ensemble of jets in an event $[10,11]$

$$
\rho=\underset{j \in \text { jets }}{\operatorname{median}}\left[\left\{\frac{p_{\mathrm{T} j}}{A_{j}}\right\}\right]
$$

In cases where the UE activity is low, for example in the $\sqrt{s}=0.9 \mathrm{TeV}$ dataset, a modification 

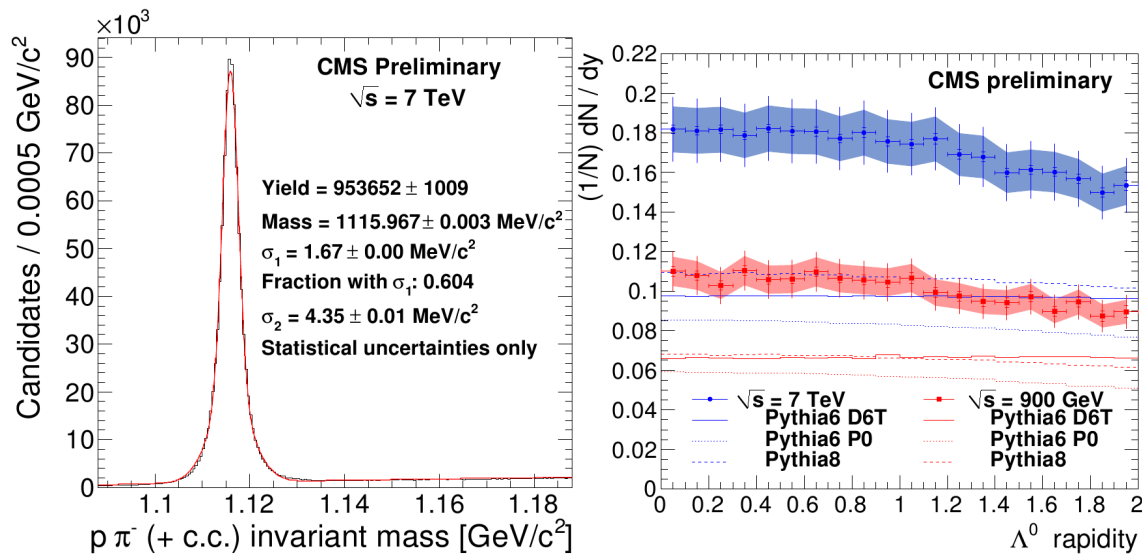

Figure 4: $\Lambda^{0}$ baryon production in NSD events. Left: Invariant mass peak. Right: Normalised rapidity distribution. The inner vertical error bars show the statistical errors, the outer the statistical and uncorrelated systematic uncertainties summed in quadrature, and the bands the overall normalisation uncertainty.

of $\rho$ variable is introduced by defining $\rho^{\prime}=\rho \cdot C$, where

$$
C=\frac{\sum_{j \in \text { physical jets }} A_{j}}{A_{\text {tot }}} .
$$

Figure 5 (left) shows the number of charged particles in the transverse region at a centre of mass energy of $7 \mathrm{TeV}$ for different Monte Carlo predictions with respect to the data. Figure 5 (right) shows the $\rho^{\prime}$ variable is plotted for several Monte Carlo predictions with respect to the data at a centre of mass energy of $0.9 \mathrm{TeV}$. The systematics and statistical uncertainties are shown in the dark and light grey bands respectively.
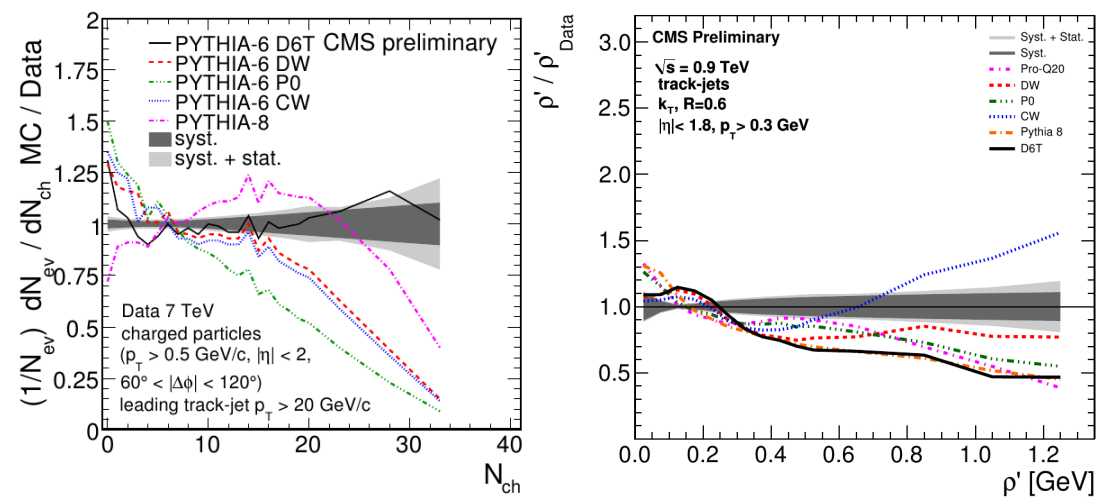

Figure 5: Underlying events measurements. Left: "Traditional" approach in terms of number of charged particles at $\sqrt{s}=7 \mathrm{TeV}$. Right: "jet area/median" approach at $\sqrt{s}=0.9 \mathrm{TeV}$.

These measurements, together with results from Spp̄S, Tevatron, and RHIC help in better 
understanding the properties of the underlying event and of multiple parton interactions in hadron-hadron scattering at high energy. This is essential for precision measurement of the Standard Model processes and for search of new physics at the LHC.

\section{Forward Processes}

A substantial fraction of the total $p p$ cross section is due to diffractive reactions of the type $p p \rightarrow X Y$ [12]. Diffractive events can be described in terms of the exchange of a colourless state with the vacuum quantum numbers and notably no colour. As a consequence, the two (groups of) final-state hadrons are well separated in rapidity ("large rapidity gap"). A diffractive signal dominated by the inclusive single-diffractive (SD) reaction $p p \rightarrow X p$ was measured and a comparison of the data with some of the available Monte Carlo models namely PYTHIA and PHOJET was carried out (figure 6). PYTHIA gives a better description of the non-diffractive component of the data, while PHOJET reproduces the diffractive contribution more accurately.
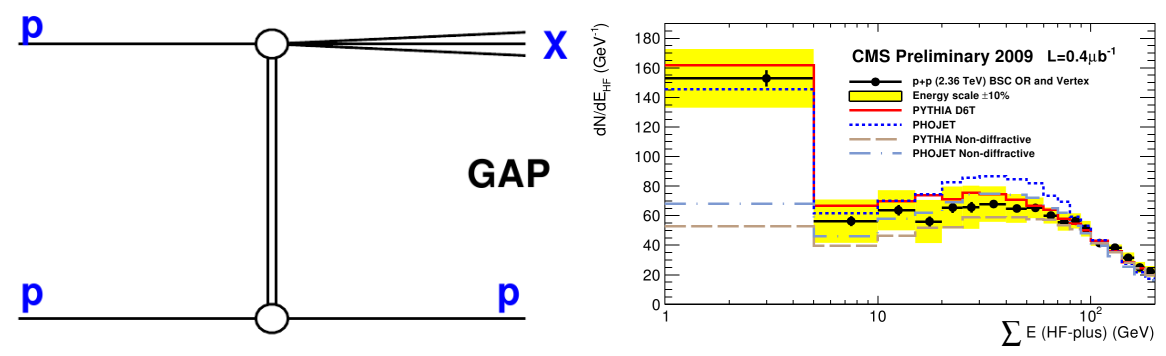

Figure 6: Left: Sketch of the SD reaction $p p \rightarrow X p$. The large rapidity gap is indicated. Right: Distribution of events as a function of the energy measured in the CMS forward hadron calorimeter at $\sqrt{s}=2.36 \mathrm{TeV}$. The predictions of PYTHIA and PHOJET are also shown, normalised to the data. The vertical bars indicate the statistical uncertainty of the data. The bands illustrate the effect of a $10 \%$ energy scale uncertainty in the calorimeters.

\section{References}

[1] CMS Collaboration, JINST 3, S08004 (2008).

[2] CMS Collaboration, Phys. Rev. Lett. 10, 26 (2010).

[3] CMS Collaboration, J. High Energy Phys. 02, 33 (2010).

[4] CMS Collaboration, CMS-QCD-10-004 (2010).

[5] T. Sjöstrand, S. Mrenna, and P. Skands, Comput. Phys. Commun. 178, 27 (2007).

[6] R. Engel and J. Ranft, Phys. Rev. D 54, 18 (1995).

[7] CMS Collaboration, CMS-QCD-10-008 (2010).

[8] CMS Collaboration, CMS-QCD-10-007 (2010).

[9] CMS Collaboration, CMS-QCD-10-010 (2010).

[10] M. Cacciari, G. P. Salam, and S. Sapeta, arXiv:0912.4926, 40 (2009).

[11] CMS Collaboration, CMS-QCD-10-005 (2010).

[12] CMS Collaboration, CMS-FWD-10-001 (2010). 